\title{
Microbiological investigation of Raphanus sativus L. grown hydroponically in nutrient solutions contaminated with spoilage and pathogenic bacteria
}

\author{
Luca Settanni $^{\text {a,*}}{ }^{\text {, Alessandro Miceli }}{ }^{\text {b }}$, Nicola Francesca ${ }^{a}$, Margherita Cruciata a , Giancarlo Moschetti ${ }^{\text {a }}$ \\ a DEMETRA Department, University of Palermo, Viale delle Scienze 4, 90128 Palermo, Italy \\ b SAGA Department, University of Palermo, Viale delle Scienze 4, 90128 Palermo, Italy
}

\section{A R T I C L E I N F O}

\section{Article history:}

Received 31 July 2012

Received in revised form 30 September 2012

Accepted 3 November 2012

Available online 20 November 2012

\section{Keywords:}

Hygienic safety

Microbial transfer

Mineral nutrient solution

Raphanus sativus L.

Soilless cultivation

Undesired bacteria

\begin{abstract}
A B S T R A C T
The survival of eight undesired (spoilage/pathogenic) food related bacteria (Citrobacter freundii PSS60, Enterobacter spp. PSS11, Escherichia coli PSS2, Klebsiella oxytoca PSS82, Serratia grimesii PSS72, Pseudomonas putida PSS21, Stenotrophomonas maltophilia PSS52 and Listeria monocytogenes ATCC $19114^{\mathrm{T}}$ ) was investigated in mineral nutrient solution (MNS) during the crop cycle of radishes (Raphanus sativus L.) cultivated in hydroponics in a greenhouse. MNSs were microbiologically analyzed weekly by plate count. The evolution of the pure cultures was also evaluated in sterile MNS in test tubes. The inoculated trials contained an initial total mesophilic count (TMC) ranging between 6.69 and $7.78 \mathrm{Log} \mathrm{CFU} / \mathrm{mL}$, while non-sterile and sterile control trials showed levels of 4.39 and $0.97 \mathrm{Log} \mathrm{CFU} / \mathrm{mL}$, respectively. In general, all inoculated trials showed similar levels of TMC in MNS during the experimentation, even though the levels of the inoculated bacteria decreased. The presence of the inoculums was ascertained by randomly amplified polymorphic DNA (RAPD) analysis applied on the isolates collected at 7-day intervals. At harvest, MNSs were also analyzed by denaturing gradient gel electrophoresis (DGGE). The last analysis, except P. putida PSS21 in the corresponding trial, did not detect the other bacteria, but confirmed that pseudomonads were present in un-inoculated MNSs. Despite the high counts detected (6.44 and 7.24 CFU/g), only C. freundii PSS60, Enterobacter spp. PSS11 and K. oxytoca PSS82 were detected in radishes in a living form, suggesting their internalization.
\end{abstract}

(c) 2012 Elsevier B.V. All rights reserved.

\section{Introduction}

Consumers are becoming more and more shrewd concerning alimentation; thus, their attention to the damages of unhealthy foods has determined an increase in the request for vegetable products in the last years. However, in many developed countries the consumption of vegetables is lower than the recommended dietary guidelines and an increase in the consumption of vegetables and fruits is strongly advised. Nevertheless, often consumers' dissatisfaction with produce quality (appearance, flavour, texture, perishability and hygienic safety) may limit their consumption (O'Beirne, 2007).

Minimally processed vegetables are creating an increase of requests for fresh produce due to their convenience of use and attractive appearance and flavour, but their benefits are offset by the rapid deterioration/short shelf-life of the products in the marketplace and the potential health hazards associated with microbial growth. Although it is somehow difficult to determine the exact route of contamination, the environment and the agricultural practices from planting through harvesting are of particular concern (Buck et al., 2003). At the pre-harvest stage, the crop management might limit or eliminate the

\footnotetext{
* Corresponding author. Tel.: + 39091 23896043; fax: + 390916515531. E-mail address: luca.settanni@unipa.it (L. Settanni).
}

risks of pathogenic microbial transference from soil to vegetables (Settanni et al., 2012).

Many factors may determine the microbial contamination also during the post-harvest manipulation (Martínez-Sánchez et al., 2006); the higher the concentrations of microorganisms the more intense the washing treatment of vegetables before consumption in the fresh-cut form needs to be. For vegetables whose edible parts grow underground, soil plays a defining role on the hygienic characteristics of the products. Soil may host several microorganisms (van Elsas et al., 2007), including bacterial species that are pathogenic for humans, such as members of the Enterobacteriaceae family (Forssten, 2009), Listeria monocytogenes (Welshimer and Donker-Voet, 1971), or Stenotrophomonas maltophilia (Bollet et al., 1995) which has emerged as an important nosocomial pathogen, especially in debilitated and immunocompromised persons (Denton and Kerr, 1998). Some species of soil origin, e.g. Pseudomonas putida (Magnuson et al., 1990), can be responsible for the spoilage of vegetable products during storage. The pathogenic species can be transferred to humans during vegetable consumption and some fresh-cut products have been linked to food-borne outbreaks (Sivapalasingam et al., 2004; DeWaal and Bhuiya, 2007).

In order to increase yield and quality, alternative agricultural systems like soilless cultivation systems (or hydroponics) consisting of growing plants in water containing mineral nutrients (nutrient 
solution) have been tested for many vegetables. Soil may be replaced by mineral or organic materials that support the roots while nutrients are diffused through the nutrient solution. In these conditions, the vegetables are cleaner and have higher sanitary quality than those from traditional (in soil) cultivation systems (Orozco et al., 2008; Selma et al., 2012) as there is no contact between soil and produce. Among soilless cultivation techniques, the floating system is a cheap and easy culture system where plants are held on panels that float on the nutrient solution.

The radish (Raphanus sativus L.) is a member of the Brassicaceae family grown and consumed throughout the world and its request is on the increase (Salerno et al., 2005). The edible part of this vegetable is the swollen root (derived from hypocotyl and upper radicle tissues) that can be harvested within four to six weeks from planting. Radishes are usually eaten raw for their crisp texture and pungent, peppery flavour and are also appreciated for its content of ascorbic acid, phenolic acids, anthocyanins and glucosinolates that can have a positive effect on consumer's health (Giusti and Wrostald, 1996; Lu et al., 2008; Jing et al., 2012).

Enterobacteria and Pseudomonas spp. have been identified within the total microbial population of radish sprouts grown in soil (Skowronek et al., 1998), confirming that radishes, if not properly treated before eating, may contribute to the spread of undesired bacteria. Islam et al. (2004) stated that a one-time application of contaminated (Salmonella) irrigation water or compost can result in the contamination of radishes and carrots well beyond their growing cycle.

The cultivation on floating panels is easy to apply to radishes and allows their production in an almost ready-to-eat form, since substrate and/or pesticide residues are absent and a single washing treatment could be enough before eating. Nevertheless, mineral nutrient solution, as irrigation water in soil, might play an important role in the pathogenic contamination of the plants. Hence, the objectives of the present study were: to investigate the survival of five species of Enterobacteriaceae family, L. monocytogenes, S. maltophilia and P. putida in mineral nutrient solution during the whole soilless crop cycle of the radish; to monitor their transference to the hypocotyl; to evaluate their viability on the radish surface at harvest; and to examine their internalization in the plants.

\section{Materials and methods}

\subsection{Plant material and microbial strains}

Radish (R. sativus L.) seeds cultivar Saxa 2 were purchased from Euroselect (Bari, Italy). Sterilized sand was used as a medium to germinate seeds and grow plantlets till full cotyledon development. The sand was moistened with deionized water and placed in a 4-cm layer in a glass tray. Radish seeds were placed on the surface, covered with a $0.5-\mathrm{cm}$ layer of sand, and kept in the dark at $20{ }^{\circ} \mathrm{C}$ until cotyledon emergence.

Citrobacter freundii PSS60, Enterobacter spp. PSS11, Escherichia coli PSS2, Klebsiella oxytoca PSS82, Serratia grimesii PSS72, P. putida PSS21 and S. maltophilia PSS52 of food origin (Todaro et al., 2011), belonging to the culture collection of the Agricultural Microbiology laboratory of DEMETRA Department - University of Palermo (Palermo, Italy), were propagated in Nutrient Broth (NB) (Difco Laboratories, Detroit, MI). Except P. putida PSS21, incubated at $20{ }^{\circ} \mathrm{C}$ for $24 \mathrm{~h}$, the other cultures were incubated at $37{ }^{\circ} \mathrm{C}$ for 24 h. L. monocytogenes ATCC $19114^{\mathrm{T}}$ was propagated in Brain Heart Infusion (BHI) (Oxoid, Basingstoke, England) at $37{ }^{\circ} \mathrm{C}$ for $24 \mathrm{~h}$.

\subsection{Experimental plan}

Ten trials were followed in this work (Fig. 1). Mineral nutrient solution (MNS), prepared using ground water treated with inverse osmosis (electrical conductivity $430 \mu \mathrm{S} / \mathrm{cm}$; pH 7.7), contained $\mathrm{NO}_{3}^{-}$
(20.00 mmol/L), $\mathrm{NH}_{4}^{+}(1.25 \mathrm{mmol} / \mathrm{L}), \mathrm{H}_{2} \mathrm{PO}_{4}^{-}(2.00 \mathrm{mmol} / \mathrm{L}), \mathrm{K}^{+}$ $(11.00 \mathrm{mmol} / \mathrm{L}), \mathrm{Ca}^{2+}(5.75 \mathrm{mmol} / \mathrm{L}), \mathrm{Mg}^{2+}(1.00 \mathrm{mmol} / \mathrm{L}), \mathrm{SO}_{4}^{2-}$ $(2.30 \mathrm{mmol} / \mathrm{L}), \mathrm{Fe}^{3+}(18 \mu \mathrm{mol} / \mathrm{L}), \mathrm{Mn}^{2+}(9 \mu \mathrm{mol} / \mathrm{L}), \mathrm{BO}_{3}^{3-}(9 \mu \mathrm{mol} / \mathrm{L})$, $\mathrm{Zn}^{2+}(3.8 \mu \mathrm{mol} / \mathrm{L})$ and $\mathrm{MoO}_{4}^{2-}(3.6 \mu \mathrm{mol} / \mathrm{L})$. The electrical conductivity (EC) of the MNS was $2.5 \mathrm{mS} / \mathrm{cm}$ and the $\mathrm{pH}$ was 6.0. Fresh microbial cultures were prepared as follows: centrifuged at $10.000 \times \mathrm{g}$ for 5 min, washed in Ringer's solution (Sigma-Aldrich, Milan, Italy) and re-suspended in the same solution till reaching an optical density (OD) of ca. 1.00, measured by 6400 Spectrophotometer (Jenway Ltd., Felsted Dunmow, UK) at $600 \mathrm{~nm}$ wavelength, which approximately corresponds to a concentration of $10^{9} \mathrm{CFU} / \mathrm{mL}$. Plastic hydroponic boxes $(13.5 \mathrm{~cm}$ long $\times 9.5 \mathrm{~cm}$ wide $\times 6.0 \mathrm{~cm}$ deep), each designed to hold 6 plants in the holes of the lid (about 468 plants $/ \mathrm{m}^{2}$ ), were sterilized by autoclaving at $121{ }^{\circ} \mathrm{C}$ for $20 \mathrm{~min}$ before filling with $650 \mathrm{~mL}$ of non-sterilized MNS. Cell suspensions (Cf, C. freundii PSS60; En, Enterobacter spp. PSS11; Ec, E. coli PSS2; Ko, K. oxytoca PSS82; Sg, Ser. grimesii PSS72; Pp, P. putida PSS21; Sm, S. maltophilia PSS52; Lm, L. monocytogenes ATCC $19114^{\mathrm{T}}$ ) were added singly till a final concentration of approximately $10^{6} \mathrm{CFU} / \mathrm{mL}$; a vigorous mixing was performed by means of a sterile pipette and the boxes were closed with a plastic cover with six holes. Two control conditions were included in the system: Ctrl, non-sterilized MNS non-inoculated; and sterilized Ctrl (SCtrl), filter (0.20- $\mu \mathrm{m}$ pore size filter, Sartorius, AubagneCedex, France) sterilized MNS non-inoculated. Two boxes were prepared for each trial and two independent experiments were performed in two consecutive days.

Ten days after sowing (end of March 2012), the radish plantlets with fully developed cotyledons were picked up and before immersion in MNS, sand was removed from the roots by washing with sterile water. Plants were then plugged into the holes of the lid and tightly fastened with non-absorbent cotton, so that the roots were below the cover and the hypocotyls and the cotyledons were above the cover. All 20 boxes of each repetition were moved to an unheated plastic greenhouse (SAGA Department - University of Palermo, Italy), where the radish plants were grown during spring 2012. The MNS was monitored daily for water consumption and weekly for EC and $\mathrm{pH}$. Hydroponic boxes were replenished with new MNS when the volume of MNS dropped below $500 \mathrm{~mL}$.

In order to evaluate the behavior in MNS without the influence of other variables, the eight pure cultures, after washing, were also inoculated (at the final concentration reported above) in sterile test tubes containing filter sterilized MNS and kept in the greenhouse for the entire radish growth cycle.

\subsection{Sample collection}

The sampling plan included both MNS and plants:

a) MNS (1 mL) was sampled, in duplicate for each trial, at the time of cell suspension addition ( $\left.\mathrm{T}_{0}\right)$ and at 7-day intervals, after thorough agitation with a sterile pipette. Each sample was collected aseptically with a portable Bunsen and a sterile pipette and transferred into a sterile test tube;

b) Plants were collected at harvest with sterile dissecting scissors and tweezers used to transfer the hypocotyls into sterile plastic bags. Two hypocotyls (one from each box) were collected from each trial for direct microbial count and two other hypocotyls were collected for the internal detection of bacteria.

The pure cultures were transferred to a sterile hood and, soon after collection (1 mL), brought back to the greenhouse.

\subsection{Microbiological analysis}

Microbiological analyses were performed after decimal serial dilution of pure cultures, MNS and radish samples in Ringer's solution. Radishes (approximately $10 \mathrm{~g}$ ) were homogenized in a stomacher (BagMixer ${ }^{\circledR} 400$, Interscience, Saint Nom, France) for 2 min at the 


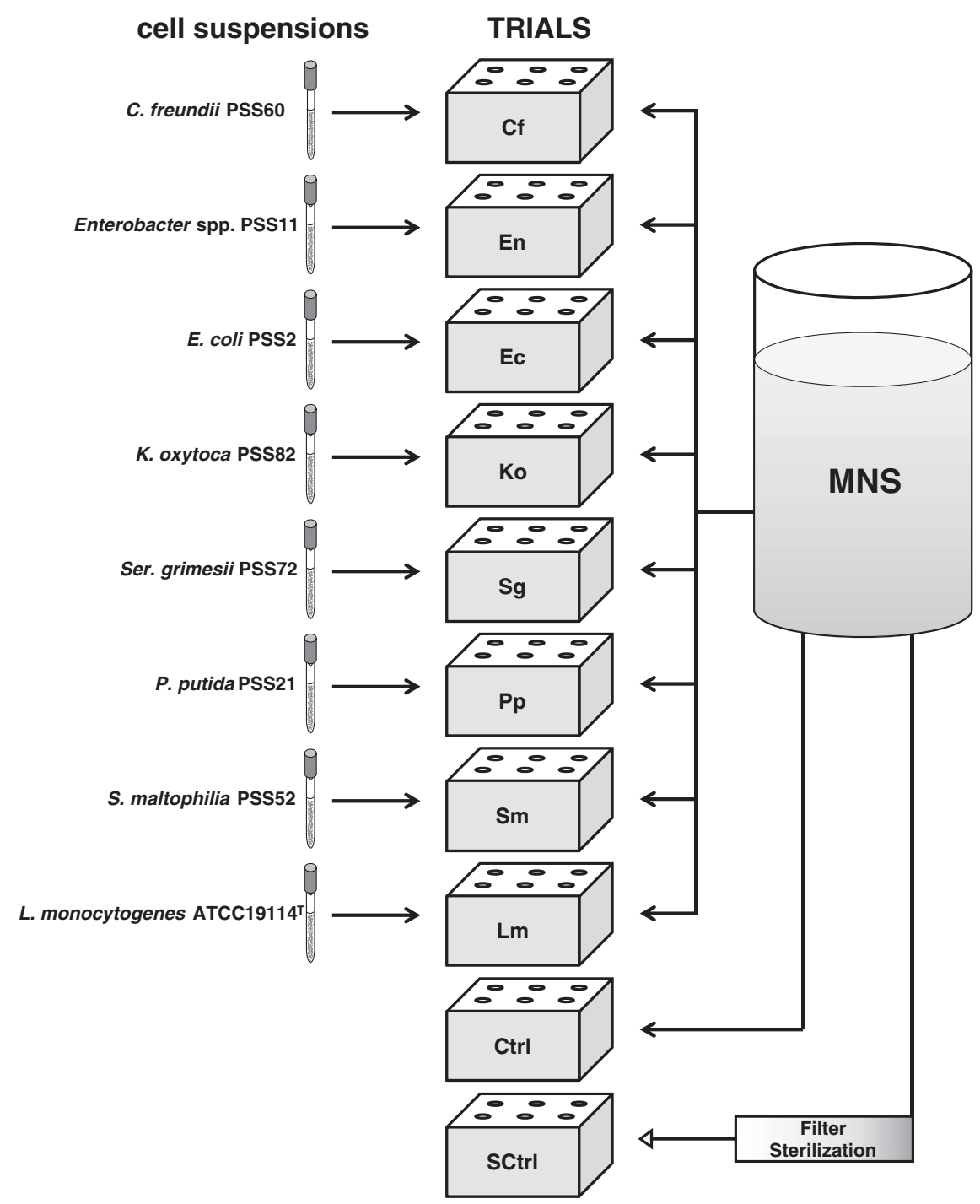

Fig. 1. Graphical representation of the inoculation scheme of mineral nutrient solution (MNS) with spoilage and pathogenic bacteria.

highest speed. Microbial suspensions were plated and incubated as follows: total mesophilic count (TMC) on Plate Count Agar (PCA), incubated aerobically at $30{ }^{\circ} \mathrm{C}$ for $72 \mathrm{~h}$ for all trials; Enterobacteria (trials Cf, En, Ec, Ko, Sg, Ctrl and SCtrl) on Violet Red Bile Glucose Agar (VRBGA) incubated aerobically at $37{ }^{\circ} \mathrm{C}$ for $24 \mathrm{~h}$; P. putida (trials $\mathrm{Pp}, \mathrm{Ctrl}$ and SCtrl) on Pseudomonas Agar Base (PAB) supplemented with $10 \mathrm{mg} / \mathrm{mL}$ cetrimide fucidin, incubated aerobically at $20^{\circ} \mathrm{C}$ for $48 \mathrm{~h}$; L. monocytogenes (trials Lm, Ctrl and SCtrl) on Listeria Selective Agar Base (LSAB) supplemented with SR140 (Oxford formulation), incubated aerobically at $37{ }^{\circ} \mathrm{C}$ for $48 \mathrm{~h}$; and S. maltophilia (trials Sm, Ctrl and SCtrl) on vancomycin-imipenem-amphotericin B (VIA) agar (Kerr et al., 1996), incubated aerobically at $30{ }^{\circ} \mathrm{C}$ for $48 \mathrm{~h}$. Microbiological counts were performed in duplicate.

All media and supplements were purchased from Oxoid.

Analysis of variance (ANOVA), elaborated with the program SPSS 14.0 (SPSS Inc., Chicago, IL, USA), was performed. Significance level $\mathrm{P}<0.05$. Tukey-Kramer's multiple range test was used to determine differences among microbial population on the plants of the different trials.

\subsection{Recognition of bacterial strains}

Several colonies developed onto the surface of the specific media (VRBGA, PAB, LSAB and VIA) at the highest dilution of MNS and hypocotyls from the inoculated trials were picked up from agar plates and cultured in NB (trials Cf, En, Ec, Ko, Sg, Pp and Sm) or BHI (trial Lm). Except cells from MNS and hypocotyls of the trial Pp, incubated overnight at $20^{\circ} \mathrm{C}$, all other cells were let to grow overnight at $37^{\circ} \mathrm{C}$. The cultures were purified to homogeneity by successive sub-culturing onto Nutrient Agar (NA) or solid BHI $(1.6 \mathrm{~g} / \mathrm{L}$ agar) in the optimal growth conditions and kept at $-80{ }^{\circ} \mathrm{C}$ in glycerol $(20 \%, \mathrm{v} / \mathrm{v})$ stocks.

The isolates and the eight pure cultures were analyzed by randomly amplified polymorphic DNA-PCR (RAPD-PCR). DNA from broth cultures was extracted by the Instagene Matrix kit (Bio-Rad, Hercules, CA) as described by the manufacturer. Crude cell extracts were used as templates for PCR reactions. RAPD-PCR was performed by means of T1 Thermocycler (Biometra, Göttingen, Germany) using the primer M13 (Stenlid et al., 1994). Amplifications were performed applying the conditions reported by Zapparoli et al. (1998). PCR products were separated by electrophoresis on $1.5 \%(\mathrm{w} / \mathrm{v}$ ) agarose gel (Gibco BRL, Cergy Pontoise, France) and visualized by UV transillumination after staining with SYBR® safe DNA gel stain (Molecular Probes, Eugene, OR). GeneRuler 100 bp Plus DNA ladder (M-Medical Srl, Milan, Italy) was used as a molecular size marker.

\subsection{Denaturing gradient gel electrophoresis (DGGE) analysis}

At harvest, MNS samples were analyzed by DGGE. One hundred milliliters of each MNS was centrifuged at $10.000 \times g$ for $5 \mathrm{~min}$ and 
the pellet was used for total DNA extraction performed with the FastDNA $®$ Pro Soil-Direct Kit (MP Biomedicals, CA, USA) following manufacturer's instructions. Bacterial DNA was amplified with the primer pair GC-HDA1/HDA2 (Walter et al., 2000). PCRs were carried out as reported by Giannino et al. (2009). The amplicons were run in polyacrilamide gels $(20 \mathrm{~cm} \times 20 \mathrm{~cm} \times 1 \mathrm{~mm})$ by means of the Phor-U2 system (Ingeny, Leiden, The Netherlands). Gels were prepared with $6 \%(\mathrm{w} / \mathrm{v})$ polyacrylamide [acrylamide/bisacrylamide $(37: 1)]$ in a $1 \times$ TAE $[40 \mathrm{mmol} / \mathrm{L}$ Tris, $20 \mathrm{mmol} / \mathrm{L}$ acetic acid, and $1 \mathrm{~mol} / \mathrm{L}$ EDTA ( $\mathrm{pH} 8.0$ )] buffer with a 30-60\% denaturing gradient [ $100 \%$ denaturant corresponding to $7 \mathrm{~mol} / \mathrm{L}$ urea and $40 \%(\mathrm{v} / \mathrm{v})$ deionised formamide] increasing in the direction of electrophoresis. Electrophoresis was performed in $1 \times \mathrm{TAE}$ at $60{ }^{\circ} \mathrm{C}$ for $14 \mathrm{~h}$. After run, gels were stained in a $\mathrm{AgNO}_{3}(0.2 \%, \mathrm{w} / \mathrm{v})$ solution (Niessen et al., 2005) and captured by Gel Doc 2000 (Bio-Rad).

\subsection{Evaluation of bacterial internalization}

In order to investigate the internal presence of the added bacterium in the hypocotyl of the corresponding trial, at harvest, two radishes for each of the eight inoculated trials were superficially sterilized. The following procedure was developed by modification of the method of Hata et al. (2002): washing with sterile distilled $\mathrm{H}_{2} \mathrm{O}\left(\mathrm{sd}-\mathrm{H}_{2} \mathrm{O}\right) ; 5$ min contact with $\mathrm{NaClO} 5 \%(\mathrm{v} / \mathrm{v})$; washing with $\mathrm{sd}-\mathrm{H}_{2} \mathrm{O} ; 90$ s contact with ethanol 95\% (v/v); washing with sd- $\mathrm{H}_{2} \mathrm{O} ; 3$ min contact with $\mathrm{H}_{2} \mathrm{O}_{2} 5 \%$ $(\mathrm{v} / \mathrm{v})$; washing with $\mathrm{sd}-\mathrm{H}_{2} \mathrm{O}$; 3 min contact with streptomycin $0.005 \%$ $(\mathrm{w} / \mathrm{v})$-cycloeximide $0.1 \%(\mathrm{w} / \mathrm{v})$; and final washing with $\mathrm{sd}-\mathrm{H}_{2} \mathrm{O}$. In order to verify the efficacy of the surface decontamination procedure applied, the treated radishes were cut into four wedges and laid (15 min) onto PCA medium so that almost the entire surface was in contact with the nutrients; petri dishes were incubated at $30{ }^{\circ} \mathrm{C}$ for $72 \mathrm{~h}$ and observed for colony development.

Surface sterile radishes were transferred into sterile bags to be homogenized after addition of the enrichment broth medium (final ratio $1: 10$ ) optimal and specific for the growth of each bacterium object of the search: Enterobacteria Enrichment Broth (EEB, OXOID) for trials Cf, En, Ec, Ko and Sg; Pseudomonas Medium (PM) (gelatine peptone $16 \mathrm{~g} / \mathrm{L}$, casein hydrolysate $10 \mathrm{~g} / \mathrm{L}$, potassium sulfate $10 \mathrm{~g} / \mathrm{L}$, magnesium chloride $1.4 \mathrm{~g} / \mathrm{L}$ ) for trial Pp; Half Fraser Broth (HFB, OXOID) for trial Lm; and VIA broth for trial Sm. Non-surface sterilized radishes were subjected to the same enrichment procedure as control for each trial. The incubation was for three days at the optimal growth temperature. The broths with a visible turbidity were streaked onto the corresponding agar media as reported above for plate counts.

The analyses were carried out in duplicate.

\section{Results}

\subsection{Radish growth}

The establishment of the plants was close to $100 \%$. After one week from transplant in the hydroponic boxes, plants started the thickening growth of the hypocotyl and after four weeks they reached the commercial size (about $2.5 \mathrm{~cm}$ on average) and were harvested. During plant growth the average temperature of air and MNS was $21.5^{\circ} \mathrm{C}$ and $23.4{ }^{\circ} \mathrm{C}$, respectively. Air temperature ranged between $32.1^{\circ} \mathrm{C}$ (day) and $11.7^{\circ} \mathrm{C}$ (night), while MNS temperature had a wider range, $38.6{ }^{\circ} \mathrm{C}$ (day) $-8.8{ }^{\circ} \mathrm{C}$ (night). The MNS used by each plant till complete hypocotyl development was about $315 \mathrm{~mL}$. Due to water absorption and evaporation and to MNS replenishing, the characteristics of MNS in the boxes varied during plant growth. The EC and pH of MNS (on average $2.93 \mathrm{mS} / \mathrm{cm}$ and 6.08 , respectively) increased slightly after two weeks of cultivation, but reached $3.52 \mathrm{mS} / \mathrm{cm}$ and 6.82 at harvest. The highest values were recorded at the fourth week, when the MNS before replenishing had an EC of $4.47 \mathrm{mS} / \mathrm{cm}$ and the $\mathrm{pH}$ was 7.40. No significant differences were found among MNS of the different trials.

\subsection{Microbiological analysis}

\subsubsection{Behavior of inoculums in sterile MNS}

The evolution of pure cultures of $C$. freundii PSS60, Enterobacter spp. PSS11, E. coli PSS2, K. oxytoca PSS82, Ser. grimesii PSS72, P. putida PSS21, S. maltophilia PSS52 and L. monocytogenes ATCC $19114^{\mathrm{T}}$ was evaluated in sterile MNS in test tubes incubated in the same environmental conditions of the radish trials (Fig. 2A-H). The initial level was in the range 6.90-7.58 Log CFU/mL. Although a low increase in concentration was registered for all eight bacteria at $24 \mathrm{~h}$ from inoculation, a general decrease in level was observed during incubation. After seven days, the concentrations were about three Log cycles lower than $\mathrm{T}_{0}$, till reaching less than $0.50 \mathrm{Log} \mathrm{CFU} / \mathrm{mL}$ for $C$. freundii PSS60 and K. oxytoca PSS82, almost 1-1.5 Log CFU/mL for the other enterobacteria and little more than $2 \mathrm{Log} C F U / \mathrm{mL}$ for the rest of the test bacteria at the 28th day of observation.

\subsubsection{Analyses of MNS}

The microbial counts of MNS during the entire growth cycle of the radish from different trials are graphically reported in Fig. 2(A-L). At the beginning of the observation, the inoculated trials contained a TMC ranging between 6.69 and $7.78 \mathrm{Log} \mathrm{CFU} / \mathrm{mL}$, while Ctrl and SCtrl showed an initial TMC of 4.39 and $0.97 \mathrm{Log}$ CFU/mL, respectively. In general, all inoculated trials showed similar levels of TMC during the 28 days of the plant growth cycle and, at the end of the period of observation, they were almost 1 Log cycle lower than those estimated at $\mathrm{T}_{0}$. Interestingly, TMC of both control trials was similar and superimposable on those displayed by the inoculated trials $(P>0.05)$ from the seventh day till the end of the experimentation.

The counts conducted with the media specific for the bacteria inoculated showed a general trend of concentration reduction. The minimum level was registered for trial Pp after 14 days from inoculation, for trials Ec and Ko at the 28th day, while for the other trials at the 21st day of experimentation. At harvest, the five trials with enterobacteria displayed concentrations of maximum 3.01 Log CFU $/ \mathrm{mL}$ onto VRBGA, while higher counts were observed for trials Pp, Sm and Lm onto PAB, VIA and LSAB, respectively. Both control trials analyzed on the same four specific media showed similar behavior onto PAB and VIA, from the beginning till the end of the radish growth, while a different observation was made onto LSAB and VRBGA. Presumptive listerias were estimated in trial Ctrl since $T_{0}$, whereas in trial SCtrl from the 14th day onward; presumptive enterobacteria ranged between $0.94\left(\mathrm{~T}_{0}\right)$ and 1.74 (28th day) Log CFU/mL in trial Ctrl, but were never detected in SCtrl. The direct comparison of data registered on the specific media from the inoculated trials with those from Ctrl and SCtrl, except in case of trial Pp, showed that the concentration levels estimated onto VRBGA, VIA and LSAB were only due to the inoculated bacteria, excluding the interference by the microorganisms present in MNS added daily. Regarding trial Pp, MNS seemed to have influenced the counts observed on PAB.

\subsubsection{Analyses of plants}

At harvest, plants were microbiologically investigated for TMC and for the presence of the bacteria inoculated in the different trials (Table 1). TMC values did not show significant differences among the different trials, including controls; they ranged between 6.44 and $7.24 \mathrm{CFU} / \mathrm{g}$. Also the counts estimated onto the specific media for the inoculated trials were not statistically different from those registered for the control trials and the highest levels of concentration (5.95-6.25 CFU/g) were registered on PAB. 
A
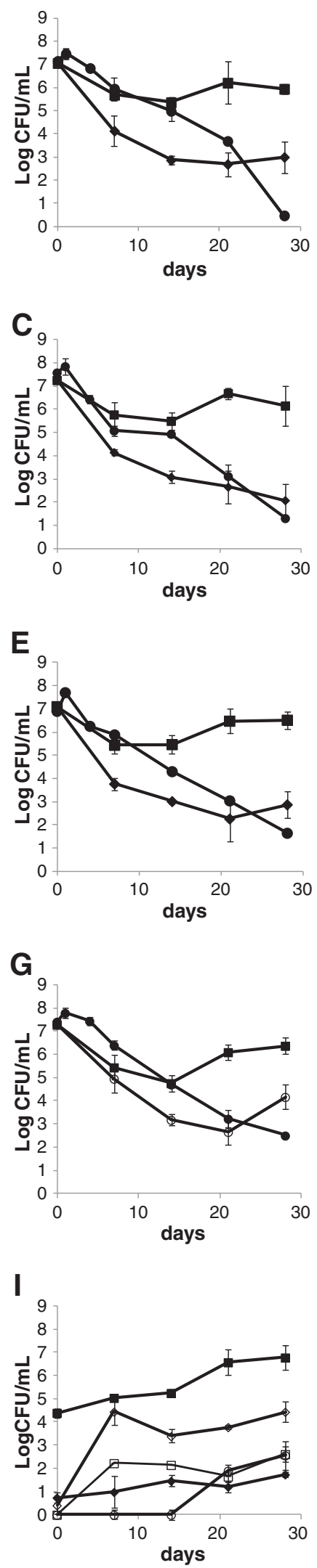

B

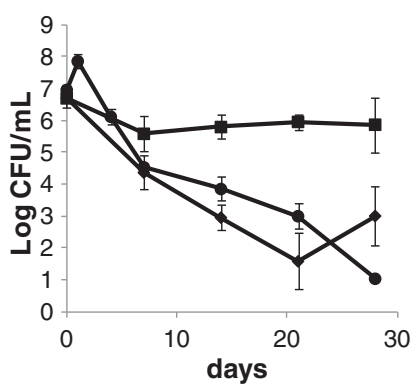

D

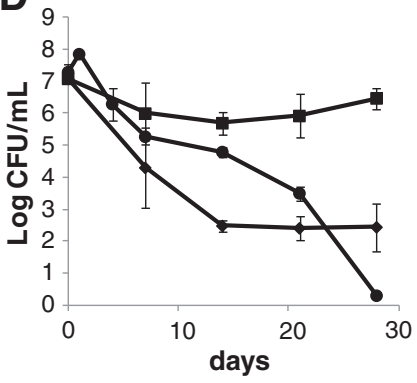

$\mathbf{F}$

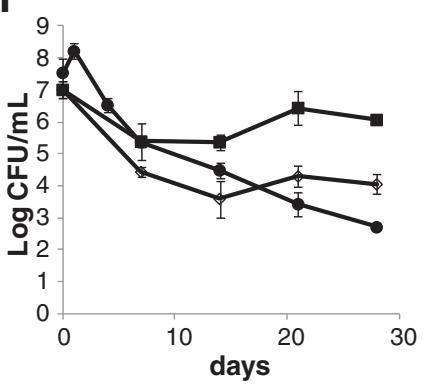

$\mathbf{H}_{9}$

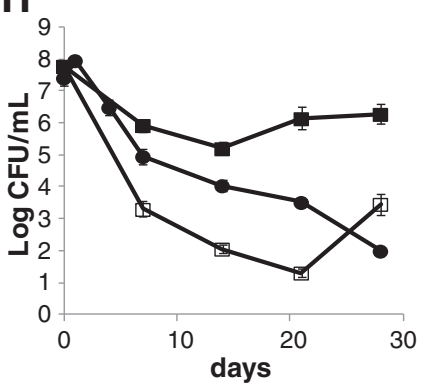

$\mathbf{L}$

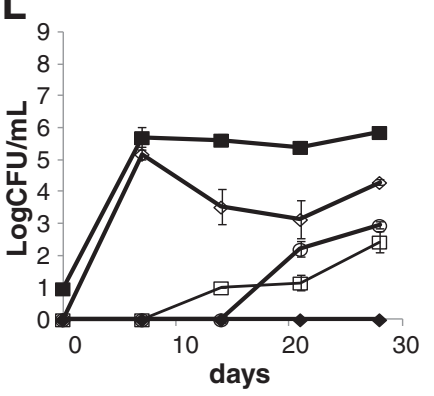

Table 1

Microbial load (Log CFU/g) of radish samples at harvest.

\begin{tabular}{llllll}
\hline \multirow{2}{*}{ Trials } & \multicolumn{2}{l}{ Media } & & & \\
\cline { 2 - 6 } & PCA & VRBGA & PAB & VIA & LSAB \\
\hline Cf & $6.86 \pm 0.28 \mathrm{a}$ & $4.55 \pm 0.22 \mathrm{a}$ & n.d. & n.d. & n.d. \\
En & $6.76 \pm 0.53 \mathrm{a}$ & $5.05 \pm 0.27 \mathrm{a}$ & n.d. & n.d. & n.d. \\
Ec & $7.24 \pm 0.28 \mathrm{a}$ & $4.81 \pm 0.26 \mathrm{a}$ & n.d. & n.d. & n.d. \\
Ko & $6.70 \pm 0.16 \mathrm{a}$ & $3.92 \pm 0.87 \mathrm{a}$ & n.d. & n.d. & n.d. \\
Sg & $6.44 \pm 0.11 \mathrm{a}$ & $4.78 \pm 0.15 \mathrm{a}$ & n.d. & n.d. & n.d. \\
Pp & $6.98 \pm 0.46 \mathrm{a}$ & n.d. & $6.25 \pm 0.22 \mathrm{a}$ & n.d. & n.d. \\
Sm & $6.78 \pm 0.22 \mathrm{a}$ & n.d. & n.d. & $4.47 \pm 0.27 \mathrm{n}$ & n.d. \\
Lm & $7.13 \pm 0.98 \mathrm{a}$ & n.d. & n.d. & n.d. & $3.57 \pm 0.42 \mathrm{a}$ \\
Ctrl & $6.61 \pm 0.48 \mathrm{a}$ & $4.08 \pm 0.75 \mathrm{a}$ & $5.95 \pm 0.82 \mathrm{a}$ & $3.85 \pm 0.66 \mathrm{a}$ & $2.57 \pm 0.81 \mathrm{a}$ \\
SCtrl & $7.23 \pm 0.20 \mathrm{a}$ & $4.06 \pm 0.91 \mathrm{a}$ & $6.39 \pm 0.07 \mathrm{a}$ & $3.82 \pm 0.83 \mathrm{a}$ & $3.13 \pm 0.18 \mathrm{a}$
\end{tabular}

Abbreviations are as follows: Cf, mineral nutrient solution (MNS) inoculated with C. freundii PSS60; En, MNS inoculated with Enterobacter spp. PSS11; Ec, MNS inoculated with E. coli PSS2; Ko, MNS inoculated with K. oxytoca PSS82; Sg, MNS inoculated with Ser. grimesii PSS72; Pp, MNS inoculated with P. putida PSS21; Sm, MNS inoculated with S. maltophilia PSS52; Lm, MNS inoculated with L. monocytogenes ATCC $19114^{\mathrm{T}}$; Ctrl, MNS non-inoculated (control trial); SCtrl sterilized MNS non-inoculated (sterilized control trial). PCA, plate count agar; VRBGA, violet red bile glucose agar; PAB, Pseudomonas agar base; VIA, vancomycin-imipenem-amphotericin B agar; and LSAB, Listeria selective agar base.

Results indicate mean value $\pm S D$ of four replicates of two independent experiments. Values within the same column followed by the same letter are not statistically different at $\mathrm{P}<0.05$ (Tukey-Kramer's multiple range test).

n.d. not determined.

\subsection{Bacterial comparison}

The isolates collected from VRBGA, PAB, LSAB and VIA resulting from plate count of MNS of the inoculated trials were analyzed at strain level by means of RAPD-PCR with primer M13. Amplified DNAs from the isolates of a given trial, together with that of the pure culture corresponding to the same trial, were loaded on a gel in order to recognize the added bacteria through the radish growth cycle and to evaluate their contribution to the plate counts. The direct comparison of the RAPD patterns allowed the recognition and monitoring of the added cultures during the entire experimentation (Fig. 3A). In all eight trials, although at decreasing levels, the inoculums were confirmed to persist in MNS in a living state till the 28th day of observation. The concentration levels estimated onto the specific media were found to be exclusively due to the inoculated bacteria. Furthermore, in case of trial Pp (Fig. 2F), although the pseudomonads vehiculated by MNS (Fig. 2I) contributed to the final concentration counted onto PAB, RAPD profile comparison clearly recognized P. putida PSS21 at the highest dilution of cell suspension.

The isolates from the plate counts of the hypocotyls, at the highest dilutions, were collected and analyzed by RAPD-PCR as reported for MNS isolates. None of the isolates of any inoculated trial were found to share the same RAPD profile of the added bacteria (Fig. 3B). These results cannot exclude the presence of the bacteria in the mature hypocotyls, but clearly showed that the test organisms were not dominant in the edible part of the radishes.

Fig. 2. Microbial counts of mineral nutrient solution (MNS). A, MNS inoculated with C. freundii PSS60 (trial Cf); B, MNS inoculated with Enterobacter spp. PSS11 (trial En); C, MNS inoculated with E. coli PSS2 (trial Ec); D, MNS inoculated with K. oxytoca PSS82 (trial Ko); E, MNS inoculated with Ser. grimesii PSS72 (trial Sg); F, MNS inoculated with P. putida PSS21 (trial Pp); G, MNS inoculated with S. maltophilia PSS52 (trial Sm); H, MNS inoculated with L. monocytogenes ATCC 19114T (trial Lm); I, MNS non-inoculated (trial Ctrl); L, sterilized MNS non-inoculated (trial SCtrl). Symbols: $\bullet$, pure culture; $\mathbf{\square}$, PCA plate count agar; $\bullet$, VRBGA, violet red bile glucose agar; $\diamond, \mathrm{PAB}$, Pseudomonas agar base; $O$, VIA, vancomycin-imipenem-amphotericin B agar; $\square$, LSAB, Listeria selective agar base Data represent the mean of four replicates of two independent experiments. Bars represent standard deviation of the mean. Vertical bars not visible are smaller than symbol size. 


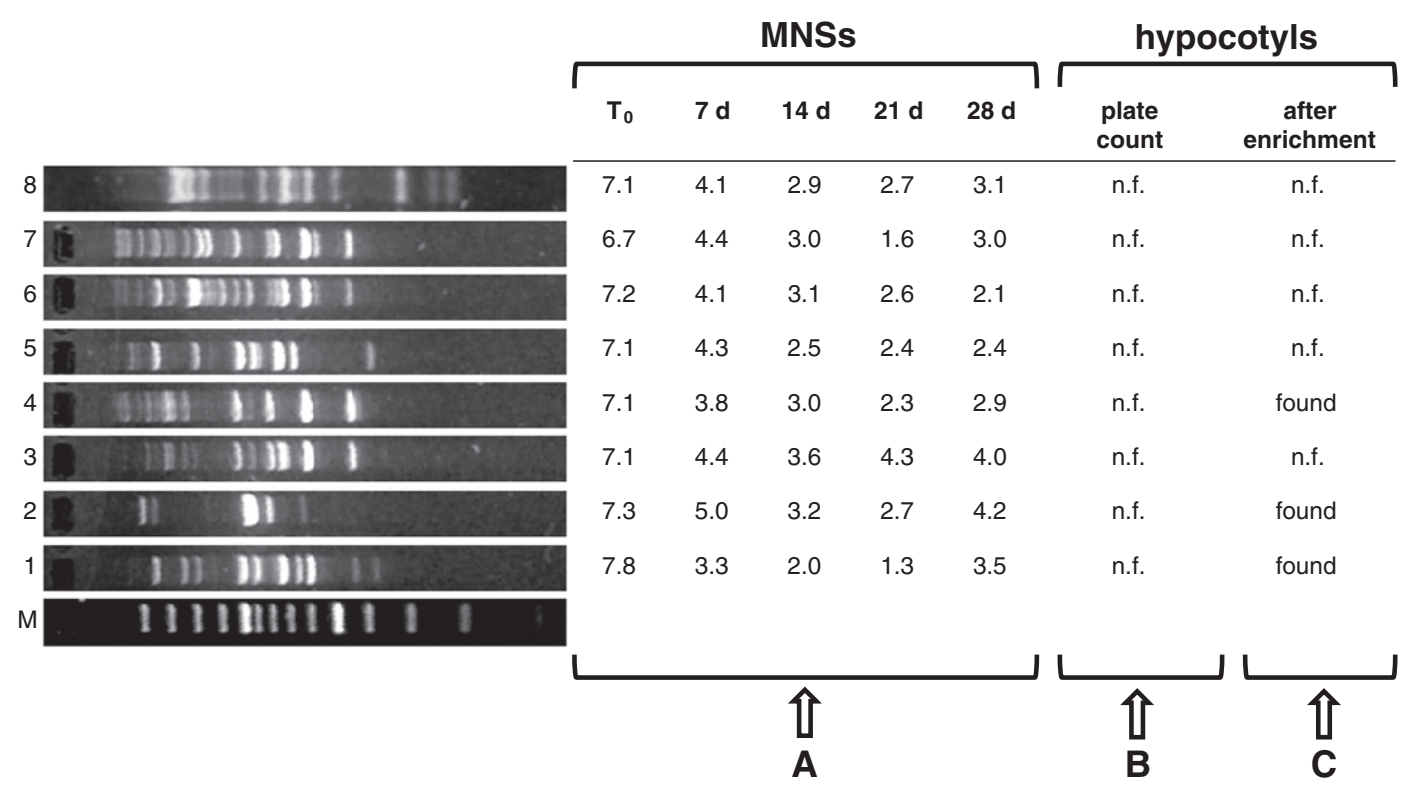

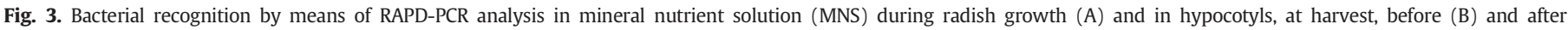

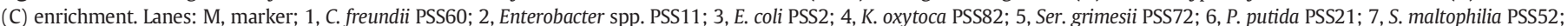
8, L. monocytogenes ATCC $19114^{\mathrm{T}}$. The maximum level of detection (Log CFU/mL) of the single inoculums in MNS is reported for each collection time. Abbreviations: n.f., not found.

\subsection{Culture-independent analysis of MNS}

DGGE analysis was applied on total DNA extracted from MNS at the time of radish harvest to better investigate the microbial communities developed in the experimental hydroponic systems. DNAs from pure cultures were also loaded onto the gel for direct comparison (Fig. 4). Only Enterobacter spp. PSS11 and L. monocytogenes ATCC $19114^{\mathrm{T}}$ were characterized by a single band, while the other six cultures produced multiple bands. DGGE clearly revealed the presence of $P$. putida PSS21 in trial Pp, at the end of experimentation, while the other seven bacteria were not detected. Moreover, the bands ascribable to Pseudomonas spp. were found in all inoculated trials, as well as in the control trials. Hence, the combination of data from DGGE and plate counts confirmed that MNS influenced the levels of count of pseudomonads.

\subsection{Analysis of internalized bacteria}

The internal presence of the added bacterium in the hypocotyls of radishes was evaluated at harvest. The radishes were surface sterilized with a protocol developed in this study. The efficacy of the surface decontamination protocol was tested by direct contact of the radish surfaces of all trials with an agar medium. The validation occurred since no colony developed after incubation.

Surface sterilized radishes were subjected to enrichment after homogenization. In these conditions, only the internal microorganisms able to grow in the specific liquid media determined the turbidity of the broths. All eight enrichment broths showed a clear microbial growth after three days of incubation and, for this reason, were subsequently streaked onto the specific agar media. The colonies developed were subjected to RAPD analysis and the comparison with the pure cultures was performed as reported above. C. freundii PSS60, Enterobacter spp. PSS11 and K. oxytoca PSS82 were clearly recognized, while the other five bacteria were not found (Fig. 3C). The same results were obtained with the non-surface sterilized radishes, confirming that E. coli PSS2, Ser. grimesii PSS72, P. putida PSS21, S. maltophilia PSS52 and L. monocytogenes ATCC $19114^{\mathrm{T}}$ were not present, at least in a living form, in the mature hypocotyls.
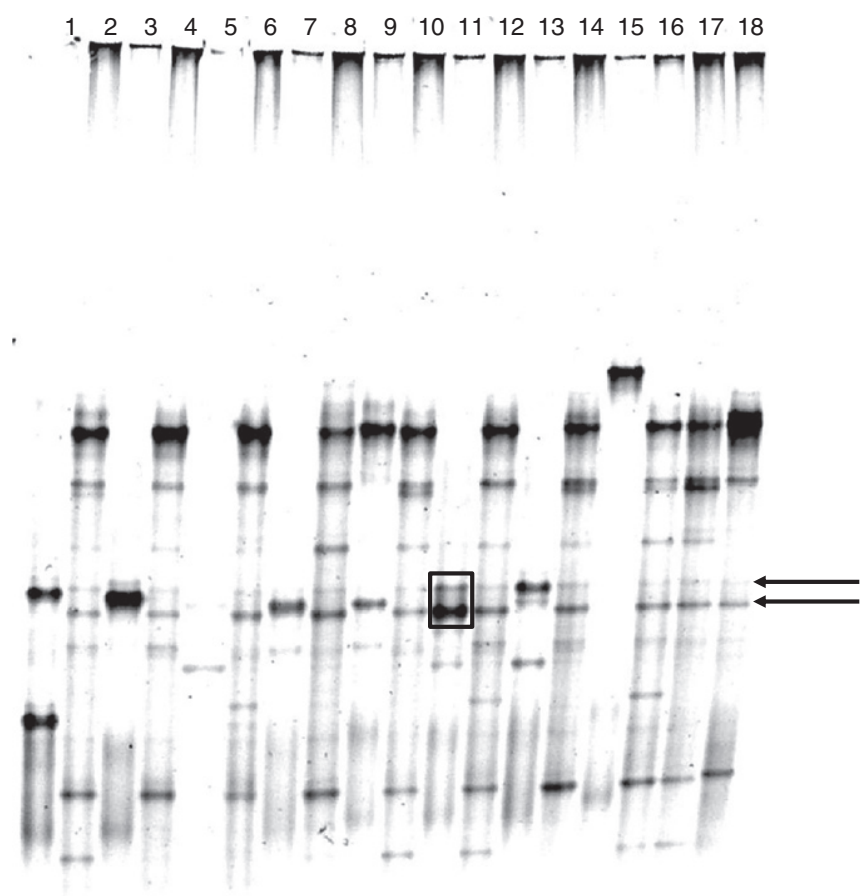

Fig. 4. Denaturing gradient gel electrophoresis (DGGE) profiles of pure cultures and mineral nutrient solution (MNS) samples at radish harvest. Lanes: 1, C. freundii PSS60; 2, trial Cf, MNS inoculated with C. freundii PSS60; 3, Enterobacter spp. PSS11; 4, trial En, MNS inoculated with Enterobacter spp. PSS11; 5, E. coli PSS2; 6, trial Ec, MNS inoculated with E. coli PSS2; 7, K. oxytoca PSS82; 8, trial Ko, MNS inoculated with K. oxytoca PSS82; 9, Ser. grimesii PSS72; 10, trial Sg, MNS inoculated with Ser. grimesii PSS72; 11, P. putida PSS21; 12, trial Pp, MNS inoculated with P. putida PSS21; 13, S. maltophilia PSS52; 14, trial Sm, MNS inoculated with S. maltophilia PSS52; 15 , L. monocytogenes ATCC $19114^{\mathrm{T}} ; 16$ trial Lm, MNS inoculated with L. monocytogenes ATCC 19114 ${ }^{\mathrm{T}} ; 17$ trial Ctrl, MNS non-inoculated (control trial); 18, trial SCtrl, sterilized MNS non-inoculated (sterilized control trial). The arrows and the box indicate the two major bands of the species $P$. putida. 


\section{Discussion}

The biological safety of foods, defined as "biosafety", refers to the analyses of the biological hazards, including bacteria, viruses, parasites, biotoxins and prions, which represent serious risks to public health. The term was coined in the 1970s after reports about the high incidence of laboratory-acquired infections among clinical and medical research laboratory staff (Pike, 1976). Good biosafety practices on farms, eradication and surveillance programs are being implemented in EU member Countries (European Commission, 2011). The effect of bacterial pathogen contamination on vegetables eaten raw has been recently recorded with $E$. coli 0124 in Northern Europe (Moulson, 2011). That event highlighted the importance of the safety of raw vegetables, especially when they are cultivated under organic regimes.

In this work, we investigated the hygienic safety of radishes grown hydroponically in MNS artificially contaminated with food spoilage and pathogenic bacteria. The levels of inoculation were around $10^{7} \mathrm{CFU} / \mathrm{mL}$ to simulate a massive contamination of MNS during the first stages of plant development. $R$. sativus was chosen not only for its short crop cycle, during which the eight spoilage/pathogen bacteria (C. freundii, Enterobacter spp., E. coli, K. oxytoca, Ser. grimesii, P. putida, S. maltophilia and L. monocytogenes), representative of several microbial groups affecting the safety and the microbiological stability of vegetables, may presumably persist at consistent concentrations, but also because it does not undergo a thermal treatment and a contamination of the produce may be dangerous for consumers. Furthermore, these plants have been tested widely in soilless cultivation on floating panels to obtain products with enhanced quality (Nicola et al., 2005; Salerno et al., 2005; Zanin et al., 2009) that need minimal sanitizing treatments before consumption.

The work was carried out with a MNS suitable for soilless cultivation of radish on floating panels. Once the plantlets were put in the boxes containing the different inoculums in MNS, they were placed in a greenhouse during the spring season, at an average air and MNS temperature of about 21.5 and $23.4{ }^{\circ} \mathrm{C}$, respectively. The plants showed fast and regular growth, and no influence of the inoculated bacteria was recorded on hypocotyls development.

In order to evaluate the influence of the microbial load of the MNS added periodically to bring the growth medium to the initial volume and the contamination by the environment, two controls (un-inoculated) were followed, one in non-sterile MNS and another one in sterile MNS. Our data showed that presumptive enterobacteria, Listeria spp. and Stenotrophomonas spp. present in MNS did not affect the counts of these microbial groups, since in both controls their concentration levels, obtained on the specific media, were lower than those registered for the inoculated trials during the entire cycle of radish growth. However, the presence of pseudomonads in Ctrl and SCtrl was in the same order of magnitude of that estimated for trial $\mathrm{Pp}$, showing a possible influence of MNS on the counts registered on PAB from the seventh day onward.

In general, all inoculated trials showed similar levels of TMC in MNS during the 28 days of the plant growth cycle, even though the counts conducted with the media specific for the inoculated bacteria decreased. At harvest, the minimum level was registered in MNS for the five trials with enterobacteria. Very few works have been conducted on the investigation of the survival of undesired food bacteria in hydroponic systems. Guo et al. (2002) found that, starting from 4.55 Log CFU/mL, the size of salmonellae in inoculated halfstrength Hoagland solution used for the cultivation of tomato plants was 4.46 to $4.65 \mathrm{Log} \mathrm{CFU} / \mathrm{mL}$ throughout the 9-day period of observation. In our study, starting from inoculation levels of about $10^{7} \mathrm{CFU} / \mathrm{mL}$ in MNS where radish roots were growing, data taken after seven days showed, for all eight bacteria object of study, levels of concentration similar to those reported by Guo et al. (2002), whereas higher levels were found for the pure cultures of the same eight organisms in sterile MNS in test tubes, a condition that excluded the interactions with the other microorganisms hosted in non-sterile MNS.

The persistence of the strains added was monitored during the experimentation by RAPD-PCR. The isolates taken from a specific medium at the highest dilution were analyzed and compared with the pure strain of the corresponding trial. The direct comparison of the RAPD patterns allowed the recognition and monitoring of the added cultures during the entire growth cycle of radish. This strategy also confirmed that, except in the case of trial Pp, the concentration levels estimated onto the specific media were exclusively due to the inoculated bacteria. The pseudomonads present in MNS contributed to the final concentration of trial Pp, but P. putida PSS21 inoculated at $\mathrm{T}_{0}$ was clearly recognized at the 28 th day of experimentation. The high counts estimated on $\mathrm{PAB}$ in both control trials since the seventh day are not surprising in this cultivation system. Fluorescent Pseudomonas species and $P$. putida were the major isolates belonging to $\gamma$-Proteobacteria in hydroponic cultures of moss Racomitrium japonicum (Tani et al., 2011). Furthermore, many Pseudomonas species were also found in the bacterial communities of the filter units employed for controlling plant pathogens in the recycled solutions used in soilless cultures (Renault et al., 2012), confirming that pseudomonads are ubiquitous in these environments. Also the finding that PAB counts in SCtrl, from 0 at $\mathrm{T}_{0}$, reached more than $10^{5} \mathrm{CFU} / \mathrm{mL}$ after seven days is not anomalous, since pseudomonads are commonly found associated to plants (Naik et al., 2008). Thus, they might have been transported to the sterile trial by plantlet roots.

DGGE analysis was useful to better evaluate data obtained with the culture-dependent methodology. Only two pure strains generated single bands, while multiple bands were displayed by the rest of the cultures employed. In general, multiple-band formation may complicate the interpretation of DGGE patterns, but in our study, this phenomenon did not interfere with the recognition of the bacterial species in MNSs. A multiple-band DGGE fingerprint for a pure strain could indicate multiple 16S rRNA gene copies with sequence divergence (Leys et al., 2004). Further molecular analyses are necessary to explain the multiple-band DGGE pattern formation of $C$. freundii PSS60, E. coli PSS2, K. oxytoca PSS82, Ser. grimesii PSS72, P. putida PSS21 and S. maltophilia PSS52.

DGGE applied on MNS at harvest showed that only the presence of $P$. putida PSS21 was clearly ascertained in trial Pp. The cultureindependent detection was not effective for the individuation of the other seven bacteria in the corresponding trials, although, as confirmed by RAPD analysis, they were isolated from the specific media. Other authors (Shinohara et al., 2011) reported that some bacteria isolated by means of dilution plating on specific media were not detected through DGGE analysis based on 16S rRNA gene, suggesting that those isolates did not constitute the major components of the microbial community investigated. In our study, TMC estimated at day 28 for MNS of the different trials was approximately $10^{6} \mathrm{CFU} / \mathrm{mL}$, while the counts detected using the specific media were no more than $10^{3} \mathrm{CFU} / \mathrm{mL}$ for enterobacteria, little more than $10^{3} \mathrm{CFU} / \mathrm{mL}$ for L. monocytogenes ATCC $19114^{\mathrm{T}}$ and reached $10^{4} \mathrm{CFU} / \mathrm{mL}$ with $P$. putida PSS21 and S. maltophilia PSS52. Since the DGGE detection limit is in the range of $1-10 \%$ of the total population (Muyzer and Smalla, 1998), both P. putida PSS21 and S. maltophilia PSS52 should have been found easily, but the latter was not detected. This result may be explained with the fact that, depending on the liquid substrate and primers employed different DGGE detection limits may be found for different bacteria (Bester et al., 2010). DGGE patterns also revealed the presence of bands ascribable to Pseudomonas spp. in all inoculated and un-inoculated trials. This finding demonstrated unequivocally that pseudomonads vehiculated by MNS influenced the levels of count detected on PAB.

Radishes were microbiologically investigated at harvest. TMC, as well as the counts estimated onto the specific media, were not statistically 
different for all trials. Thus, inoculated trials produced radishes characterized by the same levels of microbial contamination of those obtained from control trials. The levels of TMC were in the range $10^{6}-10^{7} \mathrm{CFU} / \mathrm{g}$. Our levels of TMC are different from those available in literature for radishes. Izumi (1999) reported 4.3 Log CFU/g of total microorganisms for Japanese radish shreds, raw materials that underwent a technological treatment, while Tirranen (2008) found about $8.99 \mathrm{Log}$ CFU/g of aerobic bacteria and $5.81 \mathrm{Log} \mathrm{CFU} / \mathrm{g}$ of coliforms in the near-root zone of radishes analyzed at the technical ripeness. Compared with our data, the diverse concentrations are due to the fact that those radishes were grown and collected in conditions different from those applied in the present work.

The transference of the strains added into MNS (at $\mathrm{T}_{0}$ ) was evaluated in hypocotyls (at harvest) by RAPD-PCR applied on the isolates collected from specific media at the highest dilutions. Due to the negative response of this strategy, the radishes were subsequently subjected to the enrichment procedure with and without the application of a surface sterilization treatment. In both conditions, E. coli PSS2, Ser. grimesii PSS72, P. putida PSS21, S. maltophilia PSS52 and L. monocytogenes ATCC $19114^{\mathrm{T}}$ were not found, while C. freundii PSS60, Enterobacter spp. PSS11 and K. oxytoca PSS82 were detected, showing that these bacteria were present in the internal part of hypocotyls. With this technique, the internal presence of the other five bacteria in the radishes cannot be excluded, although the results suggested that they are not present in a living form at harvest. On the other hand, the three bacteria found in radishes in living form were present at undetectable levels by plate count. Bacterial internalization in plants is a common phenomenon and it is normally a passive process. Several enteropathogens present in the irrigation water can be taken up by the root systems and enter the edible portion of different crops (Burnett et al., 2000; Guo et al., 2002; Solomon et al., 2002; Wachtel et al., 2002).

Regarding the main objectives of this work, the conclusions drafted are as follows: by means of combination of culture-dependent and culture-independent detections, the five species of Enterobacteriaceae family, L. monocytogenes, S. maltophilia and P. putida were found to survive in MNS during the whole soilless crop cycle of the radish, although at several orders of magnitude lower than inoculation; at harvest, only C. freundii PSS60, Enterobacter spp. PSS11 and K. oxytoca PSS82 were detected in the radish hypocotyls in a living form, but at subdominant levels. Since the transference of human pathogens from the cultivation system to the final product represents an issue of importance for the consumers' health, further studies on the process of internalization of the eight undesired food bacteria object of this study in radishes are being prepared, in order to picture the moment and to reveal the mechanism of their penetration, to distinguish between live and dead cells and to estimate their effective concentrations at harvest and during conservation. This study provided evidences that hydroponics are delicate cultivation systems whose contamination by undesired microorganisms, especially pathogenic species, may affect the quality of vegetables and the safety of consumers.

\section{References}

Bester, L., Cameron, M., du Toit, M., Witthuhn, R.C., 2010. PCR and DGGE detection limits for wine spoilage microbes. South African Journal of Enology and Viticulture 31, 26-33.

Bollet, C., Davin-Regli, A., De Micco, P., 1995. A simple method for selective isolation of Stenotrophomonas maltophilia from environmental samples. Applied and Environmental Microbiology 61, 1653-1654.

Buck, J.W., Walcott, R.R., Beuchat, L.R., 2003. Recent trends in microbiological safety of fruits and vegetables. Plant Health Progress http://dx.doi.org/10.1094/PHP-20030121-01-RV.

Burnett, S.L., Chen, J., Beuchat, L.R., 2000. Attachment of Escherichia coli 0157:H7 to the surfaces and internal structures of apples as detected by confocal scanning laser microscopy. Applied and Environmental Microbiology 66, 4679-4687.

Denton, M., Kerr, K.G., 1998. Microbiological and clinical aspects of infections associated with Stenotrophomonas maltophilia. Clinical Microbiology Reviews 11, 57-80.
DeWaal, C.S., Bhuiya, F., 2007. Outbreaks by the numbers: fruits and vegetables. 1990-2005 http://www.cspinet.org/foodsafety/IAFPPoster.pdf (Accessed 06.14.12).

European Commission, 2011. Green paper. The Dual-Use Export Control System of the European Union: Ensuring Security and Competitiveness in a Changing World. European Commission, Brussels . (30.06.2011 COM 393 final).

Forssten, S., 2009. Genetic basis and diagnostics of extended-spectrum $\beta$-lactamases among Enterobacteriaceae in Finland. Ph.D. Dissertation, University of Turku, Finland.

Giannino, M.L., Marzotto, M., Dellaglio, F. Feligini, M., 2009. Study of microbial diversity in raw milk and fresh curd used for Fontina cheese production by cultureindependent methods. International Journal of Food Microbiology 130, 188-195.

Giusti, M.M., Wrostald, R.E., 1996. Characterization of red radish anthocyanins. Journal of Food Science 61, 322-326.

Guo, X., Van Iersel, M.W., Chen, J., Brackett, R.E., Beuchat, L.R., 2002. Evidence of association of Salmonellae with tomato plants grown hydroponically in inoculated nutrient solution. Applied and Environmental Microbiology 68, 3639-3643.

Hata, K., Atari, R., Sone, K., 2002. Isolation of endophytic fungi from leale of Pasania edulis and their within-leaf distributions. Mycoscience 43, 369-373.

Islam, M., Morgan, J., Doyle, M.P., Phatak, S.C., Millner, P., Jiang, X., 2004. Fate of Salmonella enterica serovar Typhimurium on carrots and radishes grown in fields treated with contaminated manure composts or irrigation water. Applied and Environmental Microbiology 70, 2497-2502.

Izumi, H., 1999. Electrolyzed water as a disinfectant for fresh-cut vegetables. Journal of Food Science 64, 536-539.

Jing, P., Zhao, S.-J., Ruan, S.-Y., Xie, Z.-H., Dong, Y., Yu, L.L., 2012. Anthocyanin and glucosinolate occurrences in the roots of Chinese red radish (Raphanus sativus L.), and their stability to heat and pH. Food Chemistry 133, 1569-1576.

Kerr, K.G., Denton, M., Todd, N., Corps, C.M., Kumari, P., Hawkey, P.M., 1996. A new selective differential medium for isolation of Stenotrophomonas maltophilia. European Journal of Clinical Microbiology and Infectious Diseases 15, 607-610.

Leys, N.M.E.J., Ryngaert, A., Bastiaens, L., Verstraete, W., Top, E.M., Springael, D., 2004. Occurrence and phylogenetic diversity of Sphingomonas strains in soils contaminated with polyclyclic aromatic hydrocarbons. Applied and Environmental Microbiology 70, 1944-1955.

Lu, Z.-L., Liu, L.-W., Li, X.-Y., Gong, Y.-Q., Hou, X.-L., Zhu, X.-W., Yang, J.-L., Wang, L.-Z., 2008. Analysis and evaluation of nutritional quality in Chinese radish (Raphanus sativus L.). Agricultural Sciences in China 7, 823-830.

Magnuson, J.A., King Jr., A.D., Torok, T., 1990. Microflora of partially processed lettuce. Applied and Environmental Microbiology 56, 3851-3854.

Martínez-Sánchez, A., Allende, A., Bennett, R.N., Ferreres, F., Gil, M.I., 2006. Microbial, nutritional and sensory quality of rocket leaves as affected by different sanitizers. Postharvest Biology and Technology 42, 86-97.

Moulson, G., 2011. E. coli Death Toll up to at least 47. Associated Press.

Muyzer, G., Smalla, K., 1998. Application of denaturing gradient gel electrophoresis (DGGE) and temperature gradient gel electrophoresis (TGGE) in microbial ecology. Antonie Van Leeuwenhoek 73, 127-141.

Naik, P.R., Raman, G., Narayanan, K.B., Sakthivel, N., 2008. Assessment of genetic and functional diversity of phosphate solubilizing fluorescent pseudomonads isolated from rhizospheric soil. BMC Microbiology 8, 230.

Nicola, S., Fontana, E., Hoeberechts, J., Saglietti, D., 2005. Raphanus sativus production in soilless or traditional culture systems and postharvest packaging. Acta Horticolturae $682,1303-1310$.

Niessen, S., Mcleod, I., Yates, J.R., 2005. Identification of novel proteins complexes and protein-protein interaction by mass spectrometry. In: Golemis, E.A., Adams, P.D. (Eds.), Protein-Protein Interaction: A molecular Cloning Manual. Cold Spring Harbor Press, New York, pp. 329-354.

O'Beirne, D., 2007. Microbial safety of fresh-cut vegetables. Acta Horticulturae 746, $159-172$.

Orozco, L., Rico-Romero, L., Escartín, E.F., 2008. Microbiological profile of greenhouses in a farm producing hydroponic tomatoes. Journal of Food Protection 71, 60-65.

Pike, R.M., 1976. Laboratory-associated infections: summary and analysis of 3921 cases. Health Laboratory Science 13, 105-114.

Renault, D., Vallance, J., Deniel, F., Wery, N., Godon, J.J., Barbier, G., Rey, P., 2012. Diversity of bacterial communities that colonize the filter units used for controlling plant pathogens in soilless cultures. Microbial Ecology 63, 170-187.

Salerno, A., Pierandrei, F., Rea, E., Colla, G., Rouphael, Y., Saccardo, F., 2005. Floating system cultivation of radish (Raphanus sativus L.): production and quality. Acta Horticulturae 69, 87-92.

Selma, M.V., Luna, M.C., Martínez-Sánchez, A., Tudela, J.A., Beltrán, D., Baixauli, C., Gil, M.I., 2012. Sensory quality, bioactive constituents and microbiological quality of green and red fresh-cut lettuces (Lactuca sativa L.) are influenced by soil and soilless agricultural production systems. Postharvest Biology and Technology 63, 16-24.

Settanni, L., Miceli, A., Francesca, N., Moschetti, G., 2012. Investigation of the hygienic safety of aromatic plants cultivated in soil contaminated with Listeria monocytogenes. Food Control 26, 213-219.

Shinohara, M., Aoyama, C., Fujiwara, K., Watanabe, A., Ohmori, H., Uehara, Y., Takano, M., 2011. Microbial mineralization of organic nitrogen into nitrate to allow the use of organic fertilizer in hydroponics. Soil Science and Plant Nutrition 57, 190-203.

Sivapalasingam, S., Friedman, C.R., Cohen, L., Tauxe, R.V., 2004. Fresh produce: a growing cause of outbreaks of foodborne illness in the United States, 1973 through 1997. Journal of Food Protection 67, 2342-2353.

Skowronek, F., Simon-Sarkadi, L., Holzapfel, W.H., 1998. Hygienic status and biogenic amine content of mung bean sprouts. Zeitschrift für Lebensmittel-Untersuehung und Forschung A 207, 97-100.

Solomon, E.B., Yaron, S., Matthews, K.R., 2002. Transmission of Escherichia coli 0157:H7 from contaminated manure and irrigation water to lettuce plant tissue and its subsequent internalisation. Applied and Environmental Microbiology 68, 397-400. 
Stenlid, J., Karlsson, J.O., Hogberg, N., 1994. Intra-specific genetic variation in Heterobasidium annosum revealed by amplification of minisatellite DNA. Mycological Reseacrh 98, 57-63.

Tani, A., Akita, M., Murase, H., Kimbara, K., 2011. Culturable bacteria in hydroponic cultures of moss Racomitrium japonicum and their potential as biofertilizers for moss production. Journal of Bioscience and Bioengineering 112, 32-39.

Tirranen, L.S., 2008. Microbiota of radish plants, cultivated in closed and open ecological systems. Acta Astronautica 63, 1055-1060.

Todaro, M., Francesca, N., Reale, S., Moschetti, G., Vitale, F., Settanni, L., 2011. Effect of different salting technologies on the chemical and microbiological characteristics of PDO Pecorino Siciliano cheese. European Food Research and Technology 233, 931-940.

van Elsas, J.D., Tam, L., Finlay, R.D., Killham, K., Trevors, J.T., 2007. Microbial interactions in soil. In: van Elsas, J.D., Jansson, J.K., Trevors, J.T. (Eds.), Modern Soil Microbiology. CRC Press, Boca Raton, pp. 177-210.
Wachtel, M.R., Whitehand, L.C., Mandrell, R.E., 2002. Association of Escherichia coli 0157:H7 with preharvest leaf lettuce upon exposure to contaminated irrigation water. Journal of Food Protection 65, 18-25.

Walter, J., Tannock, G.W., Tilsala-Timisjarvi, A., Rodtong, S., Loach, D.M., Munro, K. Alatossava, T., 2000. Detection and identification of gastrointestinal Lactobacillus species by using denaturing gradient gel electrophoresis and species-specific PCR primers. Applied and Environmental Microbiology 66, 297-303.

Welshimer, H.J., Donker-Voet, J., 1971. Listeria monocytogenes in nature. Applied Microbiology 21, 516-519.

Zanin, G., Ponchia, G., Sambo, P., 2009. Yield and quality of vegetables grown in a floating system for ready-to-eat produce. Acta Horticulturae 807, 433-438.

Zapparoli, G., Torriani, S., Dellaglio, F., 1998. Differentiation of Lactobacillus sanfranciscensis strains by randomly amplified polymorphic DNA and pulsed-field gel electrophoresis. FEMS Microbiology Letters 166, 324-332. 